\title{
Holographic interpretation of 1-point toroidal block in the semiclassical limit
}

\author{
K.B. Alkalaev ${ }^{a, b}$ and V.A. Belavin ${ }^{a, c}$ \\ ${ }^{a}$ I.E. Tamm Department of Theoretical Physics, P.N. Lebedev Physical Institute, \\ Leninsky ave. 53, 119991 Moscow, Russia \\ ${ }^{b}$ Moscow Institute of Physics and Technology, \\ Dolgoprudnyi, 141700 Moscow region, Russia \\ ${ }^{c}$ Department of Quantum Physics, Institute for Information Transmission Problems, \\ Bolshoy Karetny per. 19, 127994 Moscow, Russia \\ E-mail: alkalaev@lpi.ru, belavin@lpi.ru
}

ABSTRACT: We propose the holographic interpretation of the 1-point conformal block on a torus in the semiclassical regime. To this end we consider the linearized version of the block and find its coefficients by means of the perturbation procedure around natural seed configuration corresponding to the zero-point block. From the AdS/CFT perspective the linearized block is given by the geodesic length of the tadpole graph embedded into the thermal AdS plus the holomorphic part of the thermal AdS action.

KeYwords: AdS-CFT Correspondence, Conformal and W Symmetry

ARXiv EPrint: 1603.08440 


\section{Contents}

1 Introduction 1

2 Classical 1-point toroidal block $\quad 2$

3 Dual interpretation $\quad 4$

$\begin{array}{ll}3.1 & \text { Worldline approach }\end{array}$

$\begin{array}{lll}3.2 & \text { Equilibrium condition } & 8\end{array}$

3.3 Half-cycle condition $\quad 8$

3.4 Perturbative expansion $\quad 9$

4 Conclusion $\quad 12$

$\begin{array}{ll}\text { A The linearized block coefficients } & 13\end{array}$

\section{Introduction}

The $\mathrm{AdS}_{3} / \mathrm{CFT}_{2}$ correspondence is rich and diverse subject which is in a constant state of growth. In particular, many exact results are known in the semiclassical regime where the central charge tends to infinity or, equivalently, the gravitational coupling is small [1]. In particular, it has been shown recently that classical conformal blocks on the Riemann sphere are identified with lengths of geodesic networks stretched in the asymptotically AdS space with the angle deficit or BTZ black hole [2-11].

Meanwhile, it is well known that the general solution to the classical Euclidean $\mathrm{AdS}_{3}$ gravity is topologically associated with a solid torus [12], so that the corresponding boundary CFT is essentially toroidal theory characterized by parameters of the given particular solution [13]. In this paper we are interested in toroidal conformal blocks and their dual realization. For the previous studies of the toroidal conformal blocks in the framework of CFT see [14-21].

We propose the following holographic interpretation of the linearized classical 1-point block on a torus. The bulk geometry is identified with the thermal AdS, while both intermediate and external fields of the classical block are represented by propagating massive particles with masses given by classical conformal dimensions. Note that in the toroidal case the background is not produced by fields of the 1-point function, both the external and intermediate particles are dynamical. This is in contrast with conformal blocks on the Riemann sphere appeared in the AdS/CFT context, where two heavy fields create singularities of the corresponding angle deficit/BTZ geometries. It is clear that the presence of the heavy fields in that case was aimed to produce a cylindrical topology for the boundary CFT which is appropriate for the consideration in the AdS/CFT context. 

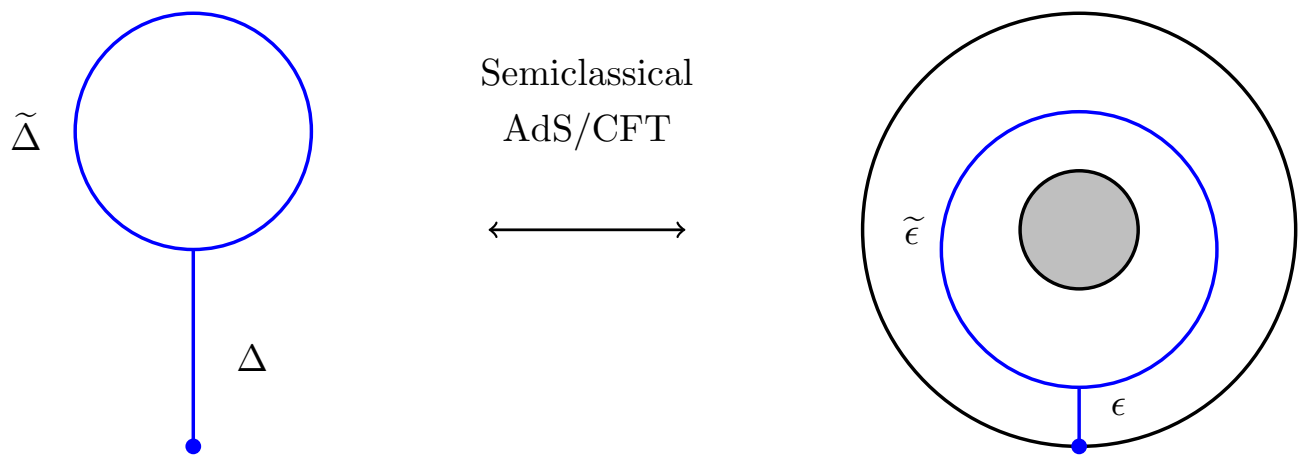

Figure 1. One-point conformal block realized as the tadpole graph embedded into the thermal AdS. The loop of the conformal block graph is identified with the non-contractible circle of the thermal AdS. $\Delta$ and $\widetilde{\Delta}$ are external and intermediate conformal dimensions, $\epsilon=k \Delta$ and $\widetilde{\epsilon}=k \widetilde{\Delta}$ are classical conformal dimensions $(k=c / 6)$.

The main result can be formulated as follows. We find that modulo regulator dependent (infinite) terms the linearized version of 1-point classical block function $f^{\text {lin }}$ with $\epsilon, \widetilde{\epsilon}$ being external and intermediate classical conformal dimensions is given by

$$
-f^{\text {lin }}=S_{\text {thermal }}+\widetilde{\epsilon} S_{\text {loop }}+\epsilon S_{\text {leg }},
$$

where the first term is the holomorphic part of the $3 \mathrm{~d}$ gravity action evaluated on the thermal AdS space, while the second and third terms give the length of the tadpole graph attached at some boundary point, see figure 1.

The outline of this paper is as follows. In section 2 we introduce the 1-point block on a torus, discuss its classical limit and then define linearized version of the block. In general, the definition requires introducing certain hierarchy of the conformal dimensions. In the case under consideration it can be described by the ratio of the external and internal conformal dimensions of the fields. We describe the series expansion of the block in terms of this parameter. In section 3 we develop the holographic interpretation. In particular, in section 3.4 we implement the perturbative procedure leading to the expansion of the geodesic length analogous to those obtained in section 2 for the conformal block. We discuss the seed geodesic configuration and its deformation, and find out that the length of the tadpole graph reproduces the conformal block function. We conclude in section 4 by discussing future perspectives. Appendix A contains the block coefficients represented as formal power series in the elliptic parameter.

\section{Classical 1-point toroidal block}

By analogy with CFT on the plane, 1-point correlation functions on the torus can be decomposed into conformal blocks (see, e.g., [16] for a review). The (holomorphic) conformal 
block of the 1-point correlator of the primary field $\phi_{\Delta}$ is given by

$$
\mathcal{V}(\Delta, \widetilde{\Delta}, c \mid q)=q^{\widetilde{\Delta}-c / 24} \sum_{n=0}^{\infty} q^{n} \mathcal{V}_{n}(\Delta, \widetilde{\Delta}, c),
$$

where

$$
q=e^{2 \pi i \widehat{\tau}},
$$

is the elliptic parameter on a torus with the modulus $\widehat{\tau}, \widetilde{\Delta}$ is the conformal dimension of the intermediate channel, and the expansion coefficients are

$$
\mathcal{V}_{n}(\Delta, \widetilde{\Delta}, c)=\frac{1}{\left\langle\widetilde{\Delta}\left|\phi_{\Delta}\right| \widetilde{\Delta}\right\rangle} \sum_{n=|M|=|N|} \frac{\left\langle\widetilde{\Delta}, M\left|\phi_{\Delta}\right| N, \widetilde{\Delta}\right\rangle}{\langle\widetilde{\Delta}, M \mid N, \widetilde{\Delta}\rangle}
$$

Here, $|\widetilde{\Delta}, M\rangle$ are the $M$-th level descendant vectors in the Verma module generated from the primary state $|\widetilde{\Delta}\rangle$. As usual, $|M|$ denotes the minus sum of Virasoro generator indices. Note that the 1-point conformal block is independent of the insertion point $z$.

One-point classical block on a torus can be defined along the same lines as in the spherical case as the leading contribution in the $c \rightarrow \infty$ approximation [21]

$$
\mathcal{V}(\Delta, \widetilde{\Delta} \mid q) \sim \exp [-k f(\epsilon, \widetilde{\epsilon} \mid q)]
$$

where $k=c / 6$, external and intermediate classical conformal dimensions $\epsilon, \widetilde{\epsilon}$ are related to the conformal dimensions by

$$
\Delta=k \epsilon, \quad \widetilde{\Delta}=k \widetilde{\epsilon},
$$

and the function $f(\epsilon, \widetilde{\epsilon} \mid q)$ is the proper classical one-point conformal block. The series expansion of this function can be easily computed by virtue of the Virasoro algebra commutation relations

$$
f(\epsilon, \widetilde{\epsilon} \mid q)=(\widetilde{\epsilon}-1 / 4) \log q+\sum_{n=1}^{\infty} q^{n} \mathrm{f}_{n}(\epsilon, \widetilde{\epsilon}),
$$

where the first few coefficients are given by

$$
\begin{aligned}
& \mathrm{f}_{1}=\frac{\epsilon^{2}}{2 \widetilde{\epsilon}}, \quad \mathrm{f}_{2}=\frac{\epsilon^{2}\left[24 \widetilde{\epsilon}^{2}(4 \widetilde{\epsilon}+1)+\epsilon^{2}(5 \widetilde{\epsilon}-3)-48 \widetilde{\epsilon}^{2}\right]}{16 \widetilde{\epsilon}^{3}(4 \widetilde{\epsilon}+3)}, \\
& \mathrm{f}_{3}=\frac{\epsilon^{2}\left[\epsilon^{4}\left(9 \widetilde{\epsilon}^{2}-19 \widetilde{\epsilon}+6\right)-16 \epsilon^{3} \widetilde{\epsilon}^{2}(7 \widetilde{\epsilon}-6)+8 \epsilon^{2} \widetilde{\epsilon}^{2}\left(60 \widetilde{\epsilon}^{2}+7 \widetilde{\epsilon}-6\right)-192 \widetilde{\epsilon} \widetilde{\epsilon}^{4}(4 \widetilde{\epsilon}+3)+48 \widetilde{\epsilon}^{4}\left(8 \widetilde{\epsilon}^{2}+10 \widetilde{\epsilon}+3\right)\right]}{48 \widetilde{\epsilon}^{5}\left(4 \widetilde{\epsilon}^{2}+11 \widetilde{\epsilon}+6\right)} .
\end{aligned}
$$

(Coefficients $\mathrm{f}_{1}$ and $\mathrm{f}_{2}$ can also be found in [21].)

The 1-point linearised classical block on a torus can be defined as in the spherical case $[6,9,22,23]$. To this end we introduce the lightness parameter

$$
\delta=\frac{\epsilon}{\widetilde{\epsilon}}<1 .
$$

Then, changing from $(\epsilon, \widetilde{\epsilon})$ to $(\delta, \widetilde{\epsilon})$ we represent the classical conformal block (2.6) as a double series expansion in $q$ and $\delta$ keeping terms at most linear in $\widetilde{\epsilon}$,

$$
f(\epsilon, \widetilde{\epsilon} \mid q)=f^{\operatorname{lin}}(\delta, \widetilde{\epsilon} \mid q)+\mathcal{O}\left(\widetilde{\epsilon}^{2}\right),
$$


where $f^{\operatorname{lin}}(\delta, \widetilde{\epsilon} \mid q)$ is the linearized classical block. Recalling the definition (2.6) we see that the linearized block is given by

$$
f^{\operatorname{lin}}(\delta, \widetilde{\epsilon} \mid q)=(\widetilde{\epsilon}-1 / 4) \log q+\widetilde{\epsilon} \sum_{n=1}^{\infty} f_{n}(q) \delta^{2 n},
$$

where the first few coefficients are given in (A.1)-(A.5) as power series in $q$. The expansion coefficients can be written in a closed form as

$$
\begin{aligned}
& f_{1}(q)=\frac{q}{2} \frac{1}{1-q}, \quad f_{2}(q)=\frac{q^{2}}{48} \frac{(q-3)}{(1-q)^{3}}, \quad f_{3}(q)=\frac{q^{3}}{480} \frac{q^{2}-5 q+10}{(1-q)^{5}} \\
& f_{4}(q)=\frac{q^{4}}{3584} \frac{q^{3}-7 q^{2}+21 q-35}{(1-q)^{7}}, \quad f_{5}(q)=\frac{q^{5}}{23040} \frac{q^{4}-9 q^{3}+36 q^{2}-84 q+126}{(1-q)^{9}} \\
& \ldots,
\end{aligned}
$$

where the general element is given by

$$
f_{n}=\frac{q^{n}}{\varkappa_{n}} \frac{q^{n-1}+\gamma_{n-2} q^{n-2}+\ldots+\gamma_{1}}{(1-q)^{2 n-1}},
$$

where $\gamma_{i}=(-)^{i}\left(\begin{array}{c}2 n+i-1 \\ i\end{array}\right), i=0,1, \ldots, n-1$ are binomial coefficients, and $\varkappa_{i}$ are some constants.

Let us note that setting $\epsilon=0$ we arrive at the 0 -point block which is the Virasoro character. It follows that the 1-point block can be considered as a small deformation of the 0-point block where the deformation parameter is identified with the external dimension. Such a deformation procedure is exactly what we have for the higher-point conformal blocks on a sphere, where $n$-point classical block was treated as a deformation of $(n-1)$-point block $[22,23]$. The procedure effectively works when $(n-1)$-point block is exactly known while the $n$-point block is not. This is the case with 1-point toroidal block, where 0 -point block is the known character.

\section{Dual interpretation}

The linearized toroidal conformal block has the holographically dual interpretation where the block function is identified with the length of the tadpole geodesic graph embedded into the three-dimensional bulk space, see figure 1 and 2. The tadpole is drawn on a two-dimensional annulus which is a slice of Euclidean thermal AdS space

$$
d s^{2}=-\tau^{2}\left(1+\frac{r^{2}}{l^{2}}\right) d t^{2}+\left(1+\frac{r^{2}}{l^{2}}\right)^{-1} d r^{2}+r^{2} d \varphi^{2}
$$

where $\tau$ is the pure imaginary modular parameter, and coordinates $t \sim t+2 \pi, \varphi \sim \varphi+2 \pi$, $r \geq 0$. Topologically, the thermal AdS is a solid torus with time running along the noncontractible cycle. In what follows, we set the AdS radius $l=1$.

Within the geodesic approximation the gravity functional integral is to be evaluated near the saddle-point given by a particular solution. It is known that in the low-temperature 


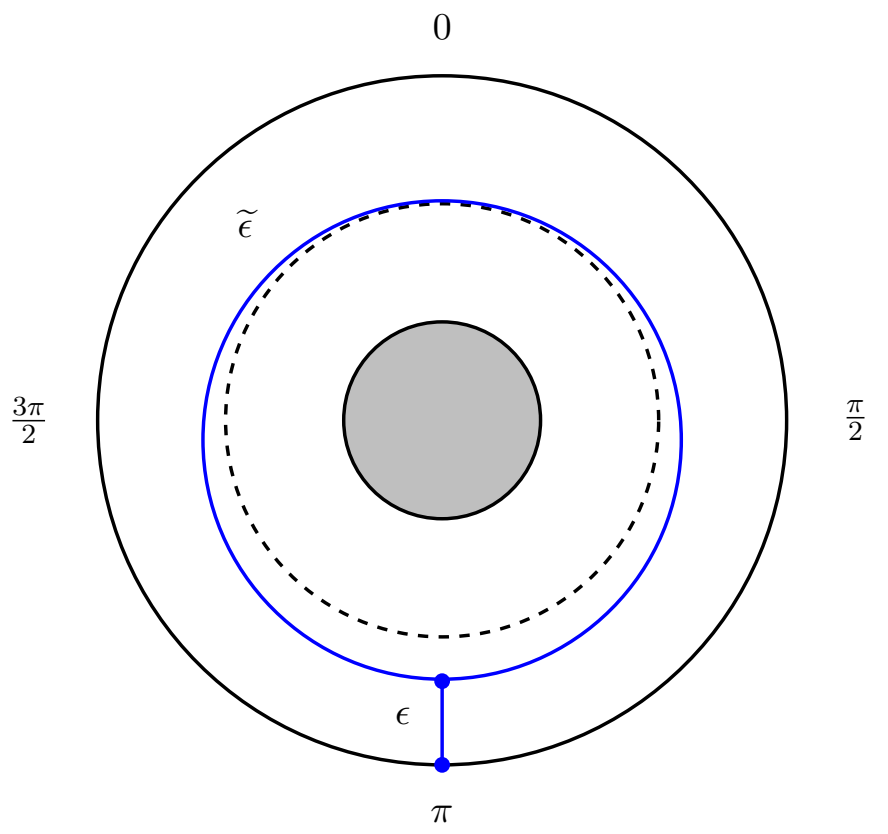

Figure 2. Annulus and tadpole graph. The inner and outer black solid circles represent the conformal boundary. The dashed circle goes along the $r=0$ radius. The blue loop is a deformation of the dashed circle when the external field represented by the solid blue segment is switched on. Vertex and boundary attachment points are at $t=\pi$. Routinely, time flows clockwise.

regime corresponding to $\operatorname{Im} \tau \gg 1$ the thermal AdS dominates the functional integral [24]. It follows that the on-shell classical action for the gravity plus the matter represented by massive external and intermediate particles is given by

$$
S_{\text {total }}=S_{\text {thermal }}+\tilde{\epsilon} S_{\text {loop }}+\epsilon S_{\text {leg }}
$$

where the first term being the gravity action on the thermal AdS, the second and third terms being the geodesic lengths of the loop and the radial line with conformal weights (2.5) identified in the $c \rightarrow \infty$ limit with masses. Below we show that that the linearized conformal block (2.10) is equal to the total action (3.2) as $f^{\text {lin }}=-S_{\text {total }}$, cf. (1.1).

In the sequel we calculate the geodesic lengths within the classical mechanics of the external and intermediate particles propagating on the surface level characterized by a constant angle. However, the spherical coordinates in (3.1) are incomplete in the sense that a loop going along the zeroth radius has no particular angle value. To complete the definition we change the parameterization of radial and angle coordinates as $-\infty<r<\infty$ and $\varphi \sim \varphi+\pi$. Then, the solid torus is represented as a stack of annuluses on figure 2 rotated along the $r=0$ circle by angles from 0 to $\pi$. The $r=0$ circle has now definite though infinitely degenerate angle position coinciding with that of the corresponding annulus.

Let us first consider the case when the external field is the identity having $\Delta=0$. Then, the 1-point conformal block becomes the 0-point conformal block identified with the Virasoro character. The corresponding graph is a loop represented by a constant radius circle going along the origin $r=0$, see figure 2. The total action (3.2) is reduced to 
$S_{\text {total }}=S_{\text {thermal }}+\tilde{\epsilon} S_{\text {loop }}$. Recalling that the holomorphic part of the gravitational action on the thermal AdS has been calculated in $[12,24,25]$ to be $S_{\text {thermal }}=i \pi \tau / 2$ in terms of the rescaled central charge $k$, while the circumference is $S_{\text {loop }}=-2 \pi i \tau$ we find that

$$
\left.S_{\text {total }}\right|_{\epsilon=0}=-2 \pi i(\widetilde{\epsilon}-1 / 4) \tau:=-(\widetilde{\epsilon}-1 / 4) \log q=-\left.f^{\operatorname{lin}}(\epsilon, \widetilde{\epsilon} \mid q)\right|_{\epsilon=0} .
$$

This is exactly the minus leading term in the classical block (2.10) provided the modular parameters are equated.

If the external field is switched on $\epsilon \neq 0$ then the constant radius circle identified with the 0-point block is deformed by the external field leg stretching from the conformal boundary to some vertex point in the bulk. ${ }^{1}$ The resulting tadpole graph corresponds to the 1-point conformal block. We assume that the loop is more massive than the external leg so in this way we implement the perturbative procedure described in the previous section to obtain the linearized conformal block. To describe the deformation we require that the radial deviation from the constant radius circle at $t=0$ is zero. Then, with time running from 0 to $\pi$, the deviation reaches its maximum value giving rise to the radial position of the vertex point where the external leg meets the loop.

Recall that in the spherical case the conical singularity/BTZ black hole is created by two heavy fields of the $n$-point correlation function. In the toroidal case the thermal AdS background is given a-priory, it is not created by any fields of the 1-point correlation function. Instead, the heavy object here is the loop while the external leg is considered as producing small deformations. (The loop can be loosely visualized as a potential line produced by two heavy fields at infinitely separated points of the cylinder but with endpoints identified.) Nonetheless, both of them are treated as probe particles propagating on the thermal AdS background. ${ }^{2}$

\subsection{Worldline approach}

The worldline approach proved to be useful in calculating lengths of various geodesic networks $[6,9,11]$. Each geodesic segment is described by the following action

$$
S=\int_{1}^{2} d \lambda \sqrt{g_{m n}(x) \dot{x}^{m} \dot{x}^{n}}
$$

where 1 and 2 are initial/final positions, local coordinates are $x^{m}=(t, \phi, r)$, the metric coefficients $g_{m n}(x)$ are read off from (3.1), and the velocity $\dot{x}$ is defined with respect to the evolution parameter $\lambda$. The action is reparametrization invariant so that one can impose the normalization condition

$$
\left|g_{m n}(x) \dot{x}^{m} \dot{x}^{n}\right|=1
$$

\footnotetext{
${ }^{1}$ In the region $\delta>1$ the loop is to be considered as a perturbation to the external leg so that in the limit $\delta=\infty$ the loop decouples from the leg because $\widetilde{\epsilon}=\epsilon / \delta$. On the CFT side, the classical block coefficients diverge in this limit, cf. (2.7).

${ }^{2}$ The configuration on figure 2 can be generalized by replacing the loop segment by a geodesic winding around the non-contractible cycle. The resulting configuration is characterized by a winding number and should lead to other local minima of the gravitational action. The dual CFT interpretation is not obvious though.
} 
allowing one to identify the on-shell value of the action as $S=\lambda_{2}-\lambda_{1}$ while the corresponding equations of motion describe geodesic curves in the thermal AdS space (3.1).

As the metric coefficients do not depend on time and angular variables it follows that the corresponding momenta are conserved, $\dot{p}_{t}=0$ and $\dot{p}_{\varphi}=0$. We restrict the dynamics to the level surface characterized by the constant angle $\varphi=0$ corresponding to conserved $p_{\phi}=0$ while the other conserved momentum $p_{t}$ is the motion integral giving the shape of a geodesics curve. The level surface is identified with the annulus resulting from slicing the thermal AdS along the non-contractible cycle. The normalization condition is then $g_{t t} \dot{t}^{2}+g_{r r} \dot{r}^{2}=1$, and taking into account $g_{t t} g_{r r}=-\tau^{2}$ we find out that

$$
\dot{r}= \pm \sqrt{r^{2}-s^{2}+1}, \quad s \equiv i \frac{\left|p_{t}\right|}{\tau} .
$$

The overall sign corresponds to the direction of the evolution parameter flow.

The circumference of the loop can be calculated by using the definition of the time momentum $p_{t}=g_{t t} \dot{t}$. Representing the loop as two semi-loops we find that

$$
\dot{t}=\frac{i}{\tau} \frac{s}{1+r^{2}}: \quad S_{\text {loop }}=\frac{2 \tau}{i s} \int_{0}^{\pi} d t\left(1+r^{2}(t)\right),
$$

where $s$ is the loop momentum, and the radial deviation $r(t)$ is defined from the normalization condition (3.6). Note that the radial deviation is the distance between the blue loop and the dashed circle shown on figure 2. It satisfies the boundary condition

$$
r(0)=0, \quad r(\pi)=\rho,
$$

where $\rho$ is the vertex radial position (see our comments below). ${ }^{3}$ If the loop is a constant radius circle then from (3.6) we find that $s^{2}=1+r^{2}$, and, therefore, $S_{\text {loop }}=-2 \pi i \tau s$. For $r=0$ the length is $S_{\text {loop }}=-2 \pi i \tau$.

The time momentum of the external leg is shown below to be vanishing, $s=0$. It means precisely that this line is stretched along the radial direction and has the length given by

$$
S_{\operatorname{leg}}=\int_{\rho}^{\Lambda} \frac{d r}{\sqrt{1+r^{2}}}=-\operatorname{ArcSinh} \rho
$$

where $\rho$ is the vertex radial position. The cutoff parameter $\Lambda$ is introduced to regularize the conformal boundary position. The integration term arising in the $\Lambda \rightarrow \infty$ limit is just an infinite constant to be discarded in the final result.

The boundary condition (3.8) is pretty well justified in the low temperature regime $\operatorname{Im} \tau \gg 1$ because in this limit the loop circumference is large. The disturbance produced by the external leg in the vertex point gradually decreases when approaching the upper point of the loop $t=0$. This intuitive estimate will be confirmed below by explicit calculations (see our comments below (3.28)).

\footnotetext{
${ }^{3}$ One can also impose less restrictive condition $r(0)=\tilde{\rho}$ implying that the loop may have non-vanishing radial deviation in the upper point. We are grateful to Per Kraus for pointing us this possibility which will be considered elsewhere.
} 


\subsection{Equilibrium condition}

The configuration of the geodesic segments near the vertex point is described by the sum of three pieces of the type (3.4). Minimizing such a combination we find that the time momenta at the vertex point (3.8) satisfy the weighted equilibrium condition

$$
\widetilde{\epsilon} p_{m}^{1}+\widetilde{\epsilon} p_{m}^{2}+\epsilon p_{m}^{0}=0
$$

where $p^{1,2}$ are the ingoing/outgoing intermediate momenta and $p^{0}$ is an external momentum at the vertex point. As the tadpole graph lies on the constant angle slice the spacetime index takes just two values $m=(t, r)$. Here, we suppose that the evolution parameter $\lambda$ is increasing away from the vertex. Obviously, any closed curve has $\left|p_{m}^{1}\right|=\left|p_{m}^{2}\right|$ while overall sings can be different. Indeed, their relative sign \pm depends on whether we take $m=t$ or $m=r$, see below.

Using parametrization (3.6) we find that the time component of (3.10) is given by

$$
\widetilde{\epsilon}\left(s_{1}-s_{2}\right)+\epsilon s_{0}=0 .
$$

As the loop has $s_{1}=s_{2} \equiv s$ we find out that $s_{0}=0$. In other words, the boundary-tobulk segment goes along the radial direction. The radial component of the equilibrium condition (3.10) is more interesting

$$
\widetilde{\epsilon}\left(\dot{r}_{1}+\dot{r}_{2}\right)-\epsilon \dot{r}_{0}=0
$$

where $\dot{r}$ are given by (3.6) with positive sign. Since $r_{1}=r_{2}$ we find that $\delta \dot{r}_{0}=2 \dot{r}_{1}$, where $\delta=\epsilon / \widetilde{\epsilon}$. Then, the vertex position $\rho$ is expressed in terms of the loop momentum $s$ as

$$
\rho=\sqrt{\frac{s^{2}}{1-\delta^{2} / 4}-1} .
$$

In particular, we see that if the external field is decoupled $\delta=0$ then there is the following solution

$$
\delta=0: \quad s=1, \quad \rho=0 .
$$

This is the seed solution for the perturbation procedure that we develop in the next sections. It means that in this case the loop is a circle, while the vertex position $\rho=0$ and $t=\pi$ is fixed to be the point where the external field leg is going to be attached to.

\subsection{Half-cycle condition}

To find how the loop radial deviation evolves in time we use the time loop momentum $p_{t}=g_{t t} \dot{t}$ and recall that $\dot{r}$ is given by (3.6). Taking their ratio we arrive at the first order differential equation

$$
\frac{d t}{d r}=\frac{i}{\tau} \frac{s}{\left(1+r^{2}\right) \sqrt{r^{2}-s^{2}+1}},
$$

where $s$ is the loop momentum. In the range $t \in[0, t]$ and $r \in[0, r]$ the above equation integrates to

$$
e^{-2 i \tau t}\left(r^{2}+1\right)\left(s^{2}-1\right)=-r\left(2 s \sqrt{r^{2}-s^{2}+1}+r s^{2}+r\right)+s^{2}-1,
$$


where the time dependence is given only via the exponential. Note that the modular parameter enters only through this exponential and this is how the elliptic parameter (2.2) appears in the final expressions. Solution (3.16) can be used to find the vertex position (3.8) through the half-cycle condition represented as

$$
e^{-2 i \pi \tau}=\frac{-\rho\left(2 s \sqrt{\rho^{2}-s^{2}+1}+\rho s^{2}+\rho\right)+s^{2}-1}{\left(\rho^{2}+1\right)\left(s^{2}-1\right)},
$$

which is given by substituting $t=\pi$ and $\rho=r(\pi)$ into (3.16). We note that values (3.14) solve the above equation.

The vertex condition (3.13) and the half-cycle condition (3.17) are two algebraic equations on two variables $\rho$ and $s$. The system can be solved unambiguously as $s=s(\delta, \tau)$ and $\rho=\rho(\delta, \tau)$. However, contrary to the vertex condition we see that the half-cycle condition is quite complicated which makes it problematic to find exact solutions. Instead, in the next section we develop the deformation method which allows one to find $s$ and $\rho$ as a power series in $\delta$ with the leading term given by the seed solution (3.14).

\subsection{Perturbative expansion}

Following the deformation method in $[9,23]$ we consider the tadpole graph perturbatively starting from the seed solution corresponding to the loop of constant radius (3.14) and adding small interaction with the external leg. In this way we are led to calculate the length function

$$
L=\widetilde{\epsilon} S_{\mathrm{loop}}(\tau, \epsilon, \widetilde{\epsilon})+\epsilon S_{\mathrm{leg}}(\tau, \epsilon, \widetilde{\epsilon}),
$$

where $S_{\text {loop }}$ and $S_{\text {leg }}$ are given by (3.7) and (3.9). It is obvious that the loop and radial segments are parameterized by the modular and conformal parameters. In particular, both lengths can be represented as power series in small parameter $\delta$ introduced in (2.8) as

$$
\begin{aligned}
S_{\text {loop }} & =\sum_{n=0}^{\infty} S_{\text {loop }}^{(n)}(\tau) \delta^{n}, \\
S_{\text {leg }} & =\sum_{n=0}^{\infty} S_{\text {leg }}^{(n)}(\tau) \delta^{n},
\end{aligned}
$$

where $S_{\text {loop }}^{(0)}(\tau)=-2 \pi i \tau$ and $S_{\text {leg }}^{(0)}(\tau)=0$. Noting that $\tilde{\epsilon} \delta^{n}=\epsilon \delta^{n-1}$ the length function is given by

$$
L=-2 \pi i \widetilde{\epsilon} \tau+\widetilde{\epsilon} \sum_{n=1}^{\infty}\left[S_{\operatorname{loop}}^{(n)}(\tau)+S_{\operatorname{leg}}^{(n-1)}(\tau)\right] \delta^{n} .
$$

Comparing with the linearized block expression (2.10) we find out that the following condition is to be satisfied

$$
S_{\text {loop }}^{(n)}(\tau)+S_{\text {leg }}^{(n-1)}(\tau)=0, \quad \text { for } \quad n=2 k+1, \quad k=0,1,2, \ldots .
$$

Below we show that this property of the expansion coefficients follows from the particular form of the time momentum and the vertex position as functions of $\delta$. 
Now, both the time momentum and the radial deviation along with the vertex position are analogously expanded as

$$
s=\sum_{n=0}^{\infty} s_{n} \delta^{n}, \quad r(t)=\sum_{n=0}^{\infty} r_{n}(t) \delta^{n}, \quad \rho=\sum_{n=0}^{\infty} \rho_{n} \delta^{n},
$$

where the seed values are $s_{0}=1, r_{0}(t)=0$ and $\rho_{0}=0$, cf. (3.8). It follows that the loop length (3.7) is

$$
\begin{aligned}
S_{\text {loop }}= & -2 \pi i \tau+2 \pi i \tau s_{1} \delta-2 i \tau \delta^{2} \int_{0}^{\pi} d t\left(r_{1}^{2}(t)+s_{1}^{2}-s_{2}\right) \\
& -2 i \tau \delta^{3} \int_{0}^{\pi} d t\left(-r_{1}^{2}(t) s_{1}+2 r_{1}(t) r_{2}(t)-s_{1}^{3}+2 s_{1} s_{2}-s_{3}\right)+\mathcal{O}\left(\delta^{4}\right) .
\end{aligned}
$$

Analogously, the radial length (3.9) is expanded as

$$
S_{\text {leg }}=-\rho_{1} \delta-\rho_{2} \delta^{2}-\left(\rho_{3}-\frac{\rho_{1}^{3}}{6}\right) \delta^{3}+\mathcal{O}\left(\delta^{4}\right) .
$$

Perturbative momentum and radius. In what follows we solve the evolution equation (3.16) and the vertex equation (3.13) perturbatively. It turns out that being expanded in $\delta$ the evolution equation contains half-integer order terms $\delta^{m / 2}$ for $m=1,2,3, \ldots$ Solving the evolution equation in this case gives rise to $0 / 0$ terms. However, all indeterminate forms can be avoided provided that the expansion (3.22) has $s_{2 k+1}=0$ and $r_{2 k}=0$ for any $k=0,1,2, \ldots$. It follows that the modified expansions read

$$
\begin{aligned}
s & =\sum_{n=0}^{\infty} s_{2 n} \delta^{2 n}=s_{0}+s_{2} \delta^{2}+s_{4} \delta^{4}+\ldots \\
r(t) & =\sum_{n=0}^{\infty} r_{2 n+1}(t) \delta^{2 n+1}=r_{1}(t) \delta+r_{3}(t) \delta^{3}+\ldots \\
\rho & =\sum_{n=0}^{\infty} \rho_{2 n+1} \delta^{2 n+1}=\rho_{1} \delta+\rho_{3} \delta^{3}+\ldots
\end{aligned}
$$

In particular, inspecting (3.23) and (3.24) we find that the condition (3.21) is now automatically satisfied.

The lowest order terms of the vertex equation and the evolution equations are given by

$$
\left(1-2 \sqrt{\rho_{1}^{2}-2 s_{2}}\right) \delta+\mathcal{O}\left(\delta^{2}\right)=0
$$

and

$$
-2 \delta^{2}\left(\left(\sqrt{r_{1}^{2}-2 s_{2}}+r_{1}\right) r_{1}+s_{2}\left(e^{-2 i \tau t}-1\right)\right)+\mathcal{O}\left(\delta^{3}\right)=0 .
$$

Solving them in each order in $\delta$ we find first radial corrections

$$
\begin{aligned}
& r_{1}(t)=\frac{1}{2} \operatorname{sech}(-i \pi \tau) \sinh (-i \tau t), \\
& r_{3}(t)=\frac{1}{16} \operatorname{sech}^{3}(-i \pi \tau) \sinh (-i \tau t) \cosh ^{2}(-i \tau t), \\
& r_{5}(t)=\frac{3}{256} \operatorname{sech}^{5}(-i \pi \tau) \sinh (-i \tau t) \cosh ^{4}(-i \tau t), \ldots,
\end{aligned}
$$


and the loop momentum corrections

$$
s_{2}=-\frac{1}{8} \operatorname{sech}^{2}(-i \pi \tau), \quad s_{4}=-\frac{1}{128} \operatorname{sech}^{4}(-i \pi \tau), \quad s_{6}=-\frac{1}{1024} \operatorname{sech}^{6}(-i \pi \tau), \ldots .
$$

The dependence on $\tau$ shows that for $\operatorname{Im} \tau \approx 1$ the radial deviation quickly starts to increase near the upper point of the loop, while for $\operatorname{large} \operatorname{Im} \tau \gg 1$ it is supported near the vertex point.

Substituting $t=\pi$ into (3.28) we find the vertex position corrections

$$
\rho_{1}=\frac{1}{2} \tanh (-i \pi \tau), \quad \rho_{3}=\frac{1}{16} \tanh (-i \pi \tau), \quad \rho_{5}=\frac{3}{256} \tanh (-i \pi \tau), \ldots .
$$

The appearance of the common factor in the above formulae allows one to represent the vertex position as

$$
\rho=g(\delta) \tanh (-i \pi \tau), \quad \text { where } \quad g(\delta)=\frac{1}{2} \delta+\frac{1}{16} \delta^{2}+\frac{3}{256} \delta^{3}+\ldots .
$$

It follows that the characteristic dependence on $\tau$ is retained while higher orders in $\delta$ appear as the overall scale factor. Also, since the modular parameter is pure imaginary the radial position is not periodic in time $t \sim t+2 \pi$. Finally, let us note that there is another branch of radial corrections with overall minus sign. It corresponds to the tadpole graph in the inner region on figure 2 .

Perturbative lengths. Using corrections (3.28), (3.29), and (3.30) it is straightforward to find corrections to the loop length and the leg length according to (3.23) and (3.24). Consider first the lowest order nontrivial corrections

$$
S_{\text {loop }}^{(2)}(\tau)=-\frac{1}{4} \tanh (-i \pi \tau), \quad S_{\text {leg }}^{(1)}(\tau)=\frac{1}{2} \tanh (-i \pi \tau) .
$$

Identifying the modular parameters as

$$
\widehat{\tau}=\tau+\frac{1}{2}
$$

we find that the above functions in the conformal parameterization are exactly

$$
S_{\text {loop }}^{(2)}(q):=f_{1}(q)+\frac{1}{4}, \quad S_{\text {leg }}^{(1)}(q):=-2 f_{1}(q)-\frac{1}{2},
$$

and so modulo an additive constant the length function (3.18), (3.20) in the first nontrivial order is

$$
L(q):=-\widetilde{\epsilon} \log q-\tilde{\epsilon} \delta^{2} f_{1}(q)+\mathcal{O}\left(\delta^{4}\right) .
$$

Adding the thermal AdS action term and comparing then with the first coefficients of the linearized classical block (2.10) we find out that $S_{\text {thermal }}(q)+L(\epsilon, \widetilde{\epsilon} \mid q)=-f(\epsilon, \widetilde{\epsilon} \mid q)$, cf. (1.1). Higher order corrections can be taken into account quite analogously. Assuming (3.33) one can show that up to multiplicative and additive constants $S_{\text {loop }}^{(2 k)} \sim f_{k}$ and $S_{\text {leg }}^{(2 k-1)} \sim f_{k}$ so that their sought-for combination is $S_{\text {loop }}^{(2 k)}+S_{\text {leg }}^{(2 k-1)}=-f_{k}+$ const $_{k}$, and the identification (1.1) holds true. 
Finally, recall that in the low-temperature regime, where the thermal AdS dominates the functional integral, the imaginary part is $\operatorname{Im}(\tau) \gg 1$ so that the real part $1 / 2$ in (3.33) can apparently be ignored. It follows that in this limit the modular parameters coincide and therefore we deal with the same torus.

\section{Conclusion}

In this paper we have proposed the AdS/CFT interpretation of the 1-point linearized classical conformal block on a torus. We have found that the block function can be calculated as the geodesic length of the tadpole graph embedded into the thermal AdS space plus the holomorphic part of the thermal AdS action.

We have seen that the correspondence holds in the low-temperature regime $\operatorname{Im} \tau \gg 1$, where the gravity functional integral is dominated by the thermal AdS. Then, the loop circumference is large so that its upper point can be treated as a point at infinity with respect to the vertex point where the leg is attached to the loop. This justifies our choice of the boundary condition (3.8) which says that the radial deviation of the deformed loop from the zeroth radius circle vanishes at the upper point. Moreover, examining the radial deviation as a function of $\tau$ we find out that the disturbance created by the external leg is indeed localized near the vertex point when $\operatorname{Im} \tau \gg 1$. Otherwise, the disturbance creates non-vanishing radial deviation already near the upper point.

On the other hand, it is known that there are other saddle points dominating the gravity functional integral at other temperatures or other $\tau$ [24]. In particular, in the high-temperature regime $\operatorname{Im} \tau \ll 1$ the thermal AdS has less action than BTZ black hole with the modular parameter related to that of the thermal AdS by the modular transformation $\tau \rightarrow-1 / \tau$. However, the worldline interpretation of the linearized classical 1-point toroidal block function $f^{\text {lin }}(\epsilon, \widetilde{\epsilon} \widehat{\tau})$ is specific to the thermal AdS and not BTZ black hole just because the leading term here is $\sim \widehat{\tau}$ rather than $\sim 1 / \widehat{\tau}$.

In particular, it explains why the toroidal CFT and the thermal AdS modular parameters cannot be equal to each other for any temperatures. Instead, they are related as in (3.33), that is the thermal AdS modulus $\tau$ is shifted by $1 / 2$ with respect to the CFT parameter $\widehat{\tau}$ while in the low-temperature approximation this difference is negligibly small. It is also worth noting that the geodesics length is a real function of real parameters so that the conformal block function should also be real. It implies that the elliptic parameter satisfies the reality condition $q=\bar{q}$ so that up to the modular equivalence there are two branches with $\operatorname{Re} \widehat{\tau}=0,1 / 2$ and arbitrary $\operatorname{Im} \widehat{\tau}$. Excluding the first branch we are naturally left with the second one realized via the holographic calculations.

Let us conclude by discussing some interesting future perspectives. The natural extension of the present results would be to develop holographic interpretation of 2-point and higher-point conformal toroidal blocks, including the $W_{N}$ symmetry case [26]. Also, it is important to study the subleading $1 / c$ corrections and related phenomena along the lines of [27-29]. The holographic consideration of various entropies in CFT on a torus is also interesting [30-32]. It would be especially important to understand the bulk worldline interpretation of the toroidal conformal blocks in terms of Wilson lines within the Chern- 
Simons formulation [33, 34], for recent discussion see, e.g., [35-38]. Finally, it is interesting to develop the geodesic Witten diagrams technique by analogy with conformal blocks on the complex plane [10]. In particular, a dual interpretation of the OPE coefficients in the toroidal case, and, more generally, how the operator algebra is realized in terms of the bulk theory is yet to be found.

\section{Acknowledgments}

We thank V. Didenko and, especially, P. Kraus for useful discussions. The work of K.A. was supported by the Russian Science Foundation grant 14-42-00047 in association with Lebedev Physical Institute. The work of V.B. was performed with the financial support of the Russian Science Foundation (Grant No.14-50-00150).

\section{A The linearized block coefficients}

The $q$-expansion of the coefficients (2.10) is given by

$$
\begin{aligned}
& f_{1}(q)=\frac{1}{2} q\left(1+q+q^{2}+q^{3}+q^{4}+q^{5}+q^{6}+q^{7}+q^{8}+q^{9}+q^{10}+\ldots\right) \\
& f_{2}(q)=-\frac{1}{48} q^{2}\left(3+8 q+15 q^{2}+24 q^{3}+35 q^{4}+48 q^{5}+63 q^{6}+80 q^{7}+99 q^{8}+120 q^{9}+\ldots\right) \\
& f_{3}(q)=\frac{1}{480} q^{3}\left(10+45 q+126 q^{2}+280 q^{3}+540 q^{4}+945 q^{5}+1540 q^{6}+2376 q^{7}+\ldots\right) \\
& f_{4}(q)=-\frac{1}{3584} q^{4}\left(35+224 q+840 q^{2}+2400 q^{3}+5775 q^{4}+12320 q^{5}+24024 q^{6}+\ldots\right) \\
& f_{5}(q)=\frac{1}{23040} q^{5}\left(126+1050 q+4950 q^{2}+17325 q^{3}+50050 q^{4}+126126 q^{5}+\ldots\right) .
\end{aligned}
$$

Open Access. This article is distributed under the terms of the Creative Commons Attribution License (CC-BY 4.0), which permits any use, distribution and reproduction in any medium, provided the original author(s) and source are credited.

\section{References}

[1] J.D. Brown and M. Henneaux, Central charges in the canonical realization of asymptotic symmetries: an example from three-dimensional gravity, Commun. Math. Phys. 104 (1986) 207 [InSPIRE].

[2] T. Hartman, Entanglement entropy at large central charge, arXiv:1303.6955 [INSPIRE].

[3] A.L. Fitzpatrick, J. Kaplan and M.T. Walters, Universality of long-distance AdS physics from the CFT bootstrap, JHEP 08 (2014) 145 [arXiv:1403.6829] [INSPIRE].

[4] C.T. Asplund, A. Bernamonti, F. Galli and T. Hartman, Holographic entanglement entropy from 2d CFT: heavy states and local quenches, JHEP 02 (2015) 171 [arXiv:1410.1392] [INSPIRE].

[5] P. Caputa, J. Simón, A. Štikonas and T. Takayanagi, Quantum entanglement of localized excited states at finite temperature, JHEP 01 (2015) 102 [arXiv:1410.2287] [INSPIRE]. 
[6] E. Hijano, P. Kraus and R. Snively, Worldline approach to semi-classical conformal blocks, JHEP 07 (2015) 131 [arXiv: 1501.02260] [INSPIRE].

[7] A.L. Fitzpatrick, J. Kaplan and M.T. Walters, Virasoro conformal blocks and thermality from classical background fields, JHEP 11 (2015) 200 [arXiv:1501.05315] [INSPIRE].

[8] E. Perlmutter, Virasoro conformal blocks in closed form, JHEP 08 (2015) 088 [arXiv: 1502.07742] [INSPIRE].

[9] K.B. Alkalaev and V.A. Belavin, Classical conformal blocks via AdS/CFT correspondence, JHEP 08 (2015) 049 [arXiv: 1504. 05943] [INSPIRE].

[10] E. Hijano, P. Kraus, E. Perlmutter and R. Snively, Semiclassical Virasoro blocks from AdS gravity, JHEP 12 (2015) 077 [arXiv:1508.04987] [INSPIRE].

[11] P. Banerjee, S. Datta and R. Sinha, Higher-point conformal blocks and entanglement entropy in heavy states, JHEP 05 (2016) 127 [arXiv: 1601.06794] [INSPIRE].

[12] S. Carlip and C. Teitelboim, Aspects of black hole quantum mechanics and thermodynamics in (2+1)-dimensions, Phys. Rev. D 51 (1995) 622 [gr-qc/9405070] [INSPIRE].

[13] A. Maloney and E. Witten, Quantum gravity partition functions in three dimensions, JHEP 02 (2010) 029 [arXiv:0712.0155] [INSPIRE].

[14] V.A. Fateev, A.V. Litvinov, A. Neveu and E. Onofri, Differential equation for four-point correlation function in Liouville field theory and elliptic four-point conformal blocks, J. Phys. A 42 (2009) 304011 [arXiv:0902.1331] [InSPIRE].

[15] R. Poghossian, Recursion relations in CFT and $N=2$ SYM theory, JHEP 12 (2009) 038 [arXiv: 0909.3412] [INSPIRE].

[16] L. Hadasz, Z. Jaskolski and P. Suchanek, Recursive representation of the torus 1-point conformal block, JHEP 01 (2010) 063 [arXiv:0911.2353] [INSPIRE].

[17] V.A. Fateev and A.V. Litvinov, On AGT conjecture, JHEP 02 (2010) 014 [arXiv: 0912.0504] [INSPIRE].

[18] P. Menotti, Riemann-Hilbert treatment of Liouville theory on the torus, J. Phys. A 44 (2011) 115403 [arXiv:1010.4946] [inSPIRE].

[19] A. Marshakov, A. Mironov and A. Morozov, On AGT relations with surface operator insertion and stationary limit of beta-ensembles, J. Geom. Phys. 61 (2011) 1203 [arXiv: 1011.4491] [INSPIRE].

[20] A.-K. Kashani-Poor and J. Troost, The toroidal block and the genus expansion, JHEP 03 (2013) 133 [arXiv:1212.0722] [INSPIRE].

[21] M. Piatek, Classical torus conformal block, $N=2^{*}$ twisted superpotential and the accessory parameter of Lamé equation, JHEP 03 (2014) 124 [arXiv:1309.7672] [INSPIRE].

[22] K.B. Alkalaev and V.A. Belavin, From global to heavy-light: 5-point conformal blocks, JHEP 03 (2016) 184 [arXiv: 1512.07627] [INSPIRE].

[23] K.B. Alkalaev and V.A. Belavin, Monodromic vs geodesic computation of Virasoro classical conformal blocks, Nucl. Phys. B 904 (2016) 367 [arXiv:1510.06685] [INSPIRE].

[24] J.M. Maldacena and A. Strominger, AdS 3 black holes and a stringy exclusion principle, JHEP 12 (1998) 005 [hep-th/9804085] [INSPIRE]. 
[25] P. Kraus, Lectures on black holes and the $A d S_{3} / C F T_{2}$ correspondence, Lect. Notes Phys. $\mathbf{7 5 5}$ (2008) 193 [hep-th/0609074] [INSPIRE].

[26] J. de Boer, A. Castro, E. Hijano, J.I. Jottar and P. Kraus, Higher spin entanglement and $\mathcal{W}_{\mathrm{N}}$ conformal blocks, JHEP 07 (2015) 168 [arXiv:1412.7520] [INSPIRE].

[27] M. Beccaria, A. Fachechi and G. Macorini, Virasoro vacuum block at next-to-leading order in the heavy-light limit, JHEP 02 (2016) 072 [arXiv:1511.05452] [INSPIRE].

[28] A.L. Fitzpatrick and J. Kaplan, Conformal blocks beyond the semi-classical limit, JHEP 05 (2016) 075 [arXiv:1512.03052] [INSPIRE].

[29] T. Anous, T. Hartman, A. Rovai and J. Sonner, Black hole collapse in the 1/c expansion, arXiv: 1603.04856 [INSPIRE].

[30] C.P. Herzog and T. Nishioka, Entanglement entropy of a massive fermion on a torus, JHEP 03 (2013) 077 [arXiv:1301.0336] [INSPIRE].

[31] S. Datta and J.R. David, Rényi entropies of free bosons on the torus and holography, JHEP 04 (2014) 081 [arXiv: 1311.1218] [INSPIRE].

[32] S. Datta, J.R. David and S.P. Kumar, Conformal perturbation theory and higher spin entanglement entropy on the torus, JHEP 04 (2015) 041 [arXiv:1412.3946] [INSPIRE].

[33] J. de Boer and J.I. Jottar, Entanglement entropy and higher spin holography in $A d S_{3}$, JHEP 04 (2014) 089 [arXiv: 1306. 4347] [INSPIRE].

[34] M. Ammon, A. Castro and N. Iqbal, Wilson lines and entanglement entropy in higher spin gravity, JHEP 10 (2013) 110 [arXiv:1306.4338] [INSPIRE].

[35] A. Hegde, P. Kraus and E. Perlmutter, General results for higher spin Wilson lines and entanglement in Vasiliev theory, JHEP 01 (2016) 176 [arXiv:1511.05555] [INSPIRE].

[36] A. Bhatta, P. Raman and N.V. Suryanarayana, Holographic conformal partial waves as gravitational open Wilson networks, JHEP 06 (2016) 119 [arXiv:1602.02962] [INSPIRE].

[37] D. Melnikov, A. Mironov and A. Morozov, On skew tau-functions in higher spin theory, JHEP 05 (2016) 027 [arXiv: 1602.06233] [INSPIRE].

[38] M. Besken, A. Hegde, E. Hijano and P. Kraus, Holographic conformal blocks from interacting Wilson lines, arXiv:1603.07317 [INSPIRE]. 